\title{
Vergamento da madeira de Eucalyptus grandis plasticizada por vaporização e cozimento
}

\author{
Solid wood bending of Eucalyptus grandis wood \\ plasticized by steam and boiling
}

\begin{tabular}{r}
\hline \\
Universidade Federal de Pelotas \\
Pelotas - RS - Brasil \\
Rafael Beltrame \\
Aceito em 29/03/15 \\
Pelotas - RS - Brasil \\
Pelotas - RS - Brasil \\
Universidade Federal de Pelotas \\
Parael de Avila Delucis \\
do Sul \\
Porto Alegre - RS - Brasil
\end{tabular}

\author{
Matheus Lemos Peres \\ Rafael de Avila Delucis \\ Darci Alberto Gatto \\ Rafael Beltrame
}

\section{Resumo}

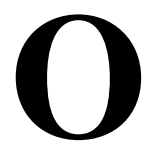

vergamento de madeira foi utilizado ao longo dos séculos para a produção de objetos grandes, como estruturas de embarcações ou pequenos utensílios. Mesmo com constantes estudos de vergamento, há carência de melhorias de processo e inclusão de madeiras de baixo valor comercial. Assim, o presente trabalho analisou a madeira vergada de Eucalyptus grandis tratada por diferentes tempos de exposição à vaporização e ao cozimento. Foram analisados o número de tipos de defeitos por peça vergada $\left(\mathrm{N}^{\mathrm{o}} \mathrm{def}\right)$, a variação no teor de umidade perante os tratamentos $(\Delta \mathrm{Tu})$, a pressão de extremidade $(\mathrm{Pe})$ e a perda de forma circular (Pf). Verificou-se que as variáveis diferem significativamente entre os tipos de tratamento de vaporização e cozimento, exceto para o $\mathrm{N}^{\circ}$ def. Os tempos de tratamento não apresentaram efeito significativo. Concluiu-se que a espécie é aceitável para vergamentos em raios inferiores ao utilizado no presente estudo. Adicionalmente, a vaporização mostrou ser o melhor tratamento, por apresentar melhores parâmetros de qualificação.

Palavras-chaves: Madeira vergada. Tratamento da madeira. Qualidade da madeira.

\section{Abstract}

Wood bending has been used for centuries in the manufacturing not only of large objects, such as boat structures, but also small objects, like musical instruments. Despite a large number of studies on bending, process improvements and inclusion of low added-value timber are still insufficient. Hence, this study proposes a bent wood analysis of Eucalyptus grandis treated with different steam and boiling exposure times. We analysed the number of failure types per sample $(N . o f f)$, moisture content variation in the treatments $(\Delta T u)$, end pressure $(P e)$ and spring back $(P f)$. The variables showed significant differences between steaming and boiling, except for N.off. Different treatment times did not show significant effects. We conclude that Eucalyptus grandis timber is suitable for bending at smaller radius than the one adopted in this study. Furthermore, steaming is better than boiling due to its higher qualification parameters.

Keywords: Wood bending. Wood treatment. Wood quality. 


\section{Introdução}

A madeira vergada sempre foi utilizada em grandes e pequenas construções, como, por exemplo, arcos para impulsionar as flechas, instrumentos musicais, estruturas de embarcações, peças decorativas e móveis (VON ZUBEN, 2010; PERES et al., 2013).

Os processos consolidados de vergamento da madeira consistem em curvá-la dentro de fôrmas com o auxílio de cintas antitração, apoios de extremidade e/ou pré-tratamentos, que têm por finalidade o alívio de tensões internas da madeira. Inicialmente, a técnica de conformação da madeira foi enfocada em seu amolecimento com água e cola quente. Nos últimos tempos, essa técnica tem sido otimizada a partir da adequação da temperatura de processo (entre $95{ }^{\circ} \mathrm{C}$ e $100{ }^{\circ} \mathrm{C}$ ) e do conteúdo de umidade (acima de $30 \%$ ), a qual atua como lubrificante para a deformação e movimentação das fibras (GAŠPARÍK; GAFF, 2013).

De modo geral, os pré-tratamentos empregados para a obtenção de peças de madeira vergada têm por essência a aplicação de temperatura em ambiente úmido, podendo ser realizados por vaporização, como também por cozimento, durante determinado período (VON ZUBEN, 2010).

A escolha da espécie a ser utilizada na manufatura de um produto de madeira sólida vergada é condicionada a sua disponibilidade, bem como à propensão à incidência de defeitos de processo. Entretanto, se for destinada para conformações mais severas, isto é, com aplicação de menores raios, deve-se primeiro considerar sua qualidade de vergamento (AYARKWA; OWUSU; APPIAH, 2011). Dessa forma, como na indústria não são comuns curvamentos severos, a madeira de Eucalyptus spp. surge como uma opção apreciável, pois sua utilização apresenta vantagens conhecidas, como cultura de manejo consolidada, crescimento rápido e grande disponibilidade de plantios.

Entretanto, embora tal gênero seja encontrado em grande volume de produção, apresenta-se em muitos casos de forma inaceitável para o vergamento, por proporcionar baixa qualidade das peças produzidas (PERES, 2011).

Portanto, é pertinente que os estudos sobre o tema sejam direcionados à elucidação das propriedades das espécies do gênero Eucalyptus quando submetidas a pré-tratamentos, ou seja, à ampliação do conhecimento a respeito de seu comportamento em relação ao vergamento. Os defeitos ocasionados na madeira em razão do procedimento de vergamento podem ser elencados como esmagamento, dobra transversal, cisalhamento longitudinal, fendas transversais, estilhaço, ruptura perpendicular e fendas de secagem (GATTO et al., 2008; PERES et al., 2013).

Nesse contexto, os parâmetros já utilizados para caracterizar a qualidade de uma peça vergada podem ser elencados como a perda de forma circular e a pressão de extremidade (SCHLEINING, 2001); a extensão do dano observado no lado côncavo e o plano anatômico de vergamento (HWANG et al., 2002); o número de defeitos unitários (PERES, 2011); o mínimo raio de curvatura e a massa específica residual (DAIAN; OZARSKA, 2010); e a percentagem de peças não quebradas (AYARKWA; OWUSU; APPIAH, 2011).

Assim, o presente trabalho teve como objetivo avaliar a qualidade de vergamento para a madeira de Eucalyptus grandis Hill ex Maiden submetida a pré-tratamentos de vaporização e cozimento.

\section{Material e métodos}

Para o estudo, foi escolhida a madeira de Eucalyptus grandis com cerca de 30 anos de idade, proveniente da cidade de Telêmaco Borba, PR (29¹9’30.01'S 50³7’05.04”O), sem vestígios de ataques de organismos xilófagos, livre de defeitos anatômicos ou inconformidades originárias do procedimento de desdobro. A madeira foi obtida em forma de tábuas, com as dimensões de $2,5 \mathrm{~cm} \mathrm{x}$ $12 \mathrm{~cm} \quad x \quad 270 \mathrm{~cm}$ (radial $\mathrm{x}$ tangencial $\mathrm{x}$ longitudinal). $\mathrm{O}$ material foi condicionado em câmara climatizada com $65 \%$ de umidade relativa do ar e $20^{\circ} \mathrm{C}$ de temperatura, até a obtenção de um teor de umidade de $12 \%$. Dessas tábuas, foram seccionadas amostras orientadas longitudinalmente com dimensões de $1,7 \mathrm{~cm}$ x $5,0 \mathrm{~cm}$ x $100 \mathrm{~cm}$ (radial $\mathrm{x}$ tangencial $\mathrm{x}$ longitudinal). A massa específica média da madeira utilizada foi de 0,47 g. $\mathrm{cm}^{-3}$ (desvio padrão: 0,066; coeficiente de variação: 13,8\%).

Para o pré-tratamento da madeira, foram utilizadas duas caixas de metal independentes, para o cozimento e a vaporização, ambas com aberturas para que fosse mantida a pressão atmosférica durante o processo. Os materiais de constituição dessas caixas foram aço inoxidável e aço zincado revestido com chapas de MDF (medium density fiberboard) respectivamente.

A Tabela 1 apresenta a relação dos pré-tratamentos utilizados, bem como a distinção entre os fatores tempo e tipo de tratamento. As amostras foram inseridas em grupos na caixa de tratamento (3 amostras por grupo), devidamente identificadas e

170 Peres, M. L.; Delucis, R. de A.; Gatto, D. A.; Beltrame, R. 
com escalonamento de tempo, para que permanecessem o mesmo período sob a ação do pré-tratamento $( \pm 3 \mathrm{~min})$. Considerando que os pré-tratamentos de cozimento e vaporização são comuns em natureza física, isto é, utilizam água ao ponto de ebulição e pressão atmosférica, a temperatura manteve-se constante a $100( \pm 2){ }^{\circ} \mathrm{C}$ para todos os grupos.

O dispositivo utilizado para o vergamento (Figuras 1 e 2) dispôs de cinta antitração, confeccionada em aço inoxidável com a espessura de $0,8 \mathrm{~mm}$, contando com apoios de extremidade (end blocks) em madeira e de aço, e uma forma com raio de curvatura de $34 \mathrm{~cm}$. A capacidade do dispositivo é de uma amostra, sendo ensaios feitos de forma individual.

Para a determinação do teor de umidade, após o ensaio de vergamento, as amostras foram secas em estufa laboratorial a uma temperatura de $103 \pm 2$ ${ }^{\circ} \mathrm{C}$ até obterem massa constante. Determinou-se a variação no teor de umidade proporcionada pelos tratamentos, referida como $\Delta \mathrm{Tu}$. Para tanto, os teores de umidade anteriores e posteriores aos tratamentos foram verificados de acordo com o recomendado pela normativa D143 - 94 (AMERICAN..., 2007). As massas das amostras foram mensuradas nos instantes anterior $(\mathrm{m} 12 \%) \mathrm{e}$ posterior (mPÓS) ao tratamento, bem como após a secagem (ma). A variável $\Delta \mathrm{Tu}$ foi obtida pela diferença nos teores de umidade inicial $\left(\mathrm{Tu}_{\mathrm{ANTES}}\right) \mathrm{e}$ final (Túpós) (Equacões 1, 2 e 3).

$$
\begin{aligned}
& \mathrm{Tu}_{\mathrm{ANTES}}=\frac{\left(\mathrm{m}_{12 \%}-\mathrm{ma}\right)}{\mathrm{ma}} \cdot 100 \\
& \mathrm{Tu}_{\mathrm{PÓS}}=\frac{\left(\mathrm{m}_{\text {Pós }}-\mathrm{ma}\right)}{\mathrm{ma}} \cdot 100 \\
& \Delta \mathrm{Tu}=\frac{\left(\mathrm{Tu} u ́ o ́_{\mathrm{S}}-\mathrm{Tu}_{\mathrm{ANTES}}\right)}{\mathrm{Tu}_{\mathrm{ANTES}}} \cdot 100
\end{aligned}
$$

Eq. 3

Onde:

$\mathrm{m}_{12 \%}=$ massa a $12 \%$ de umidade $(\mathrm{g})$;

$\mathrm{ma}=$ massa $\operatorname{anidra}(\mathrm{g})$;

$\mathrm{m}_{\mathrm{PÓs}}=$ massa após o tratamento $(\mathrm{g})$;

$\mathrm{Tu}_{\mathrm{ANTES}}=$ teor de umidade antes do tratamento $(\%)$; e

$\mathrm{Tu}_{\mathrm{PÓs}}=$ teor de umidade após o tratamento $(\%)$.

Tabela 1 - Pré-tratamentos de plasticização utilizados para o vergamento da madeira de Eucalyptus grandis

\begin{tabular}{cccc}
\hline Descrição & $\mathbf{N}^{\mathbf{0}}$ de Amostras & Tipo de Tratamento & Tempo (min) \\
\hline T1 & 10 & Vaporização & 45 \\
T2 & 10 & Vaporização & 60 \\
T3 & 10 & Vaporização & 90 \\
T4 & 10 & Vaporização & 120 \\
T5 & 10 & Cozimento & 45 \\
T6 & 10 & Cozimento & 60 \\
T7 & 10 & Cozimento & 90 \\
T8 & 10 & Cozimento & 120 \\
\hline
\end{tabular}

\section{Figura 1 - Representação gráfica do dispositivo de vergamento}

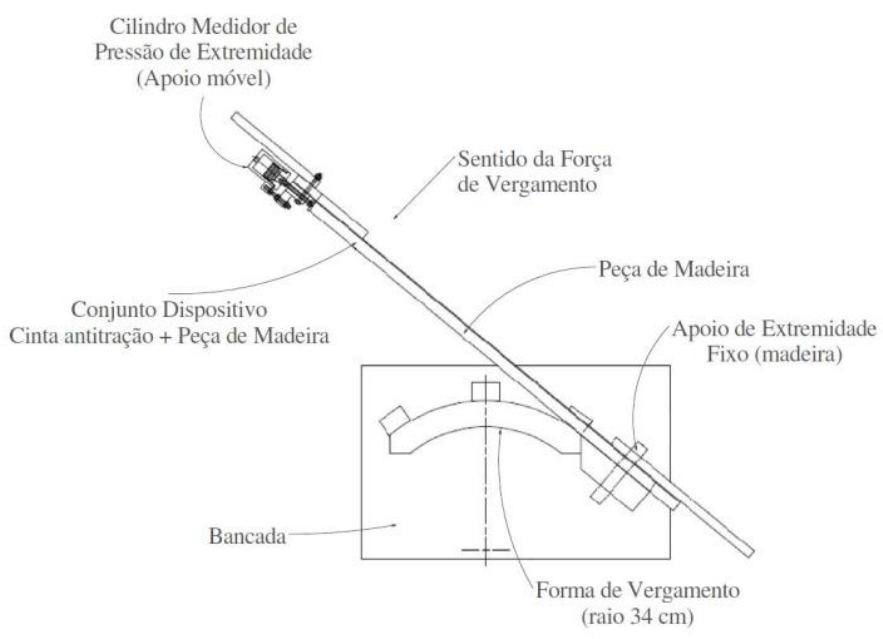

Fonte: adaptado de Von Zuben (2010). 
Figura 2 - Corpo de prova vergado fixo com o auxílio de grampos junto ao dispositivo

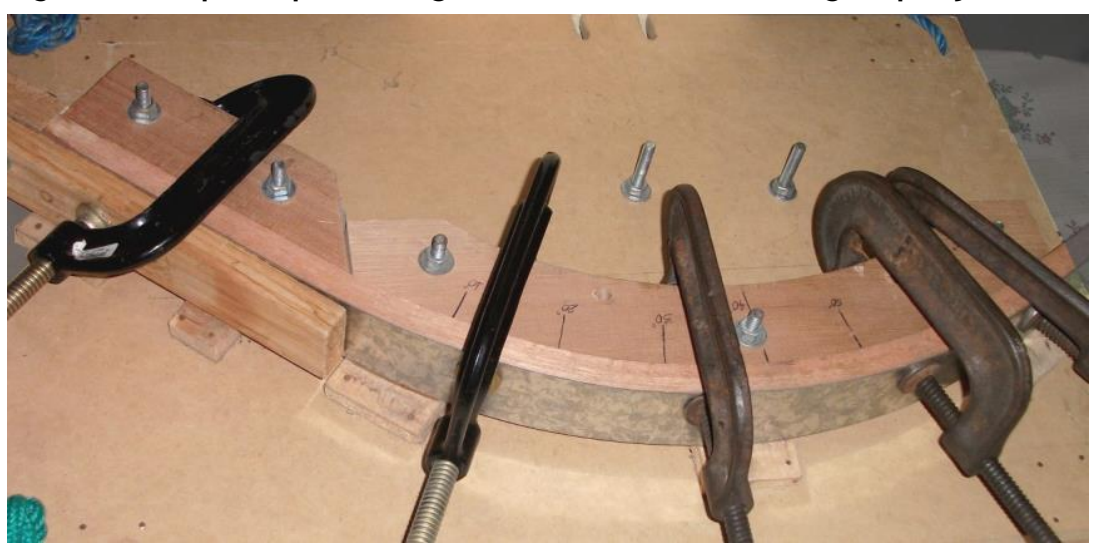

A pressão de extremidade foi avaliada por meio de um cilindro hidráulico com manômetro, posicionado em um dos apoios de extremidade, durante a aplicação da força de vergamento. O valor de leitura do manômetro foi corrigido em função da área da seção transversal do corpo de prova, para que denotasse a pressão exercida sobre a madeira (Equação 4).

$\mathrm{Pe}=\frac{\mathrm{P}_{\mathrm{m}} \cdot \mathrm{A}_{\mathrm{e}}}{\mathrm{A}_{\mathrm{CP}}}$

Onde:

$\mathrm{Pe}=$ pressão de extremidade $\left(\mathrm{kgf} . \mathrm{cm}^{-2}\right)$;

$\mathrm{P}_{\mathrm{m}}=$ pressão na madeira $\left(\mathrm{kgf.} \mathrm{cm}^{-2}\right)$;

$\mathrm{A}_{\mathrm{e}}=$ área do êmbolo $\left(\mathrm{cm}^{2}\right)$; e

$\mathrm{A}_{\mathrm{CP}}=$ área da seção transversal do corpo de prova $\left(\mathrm{cm}^{2}\right)$.

Para a avaliação da fixação da forma curvada da peça (perda de forma circular), as amostras foram preservadas no dispositivo por aproximadamente $45 \mathrm{~min}$, até seu resfriamento. Para o cálculo da perda de forma circular utilizou-se a Equação 5.

$\mathrm{Pf}=\frac{\mathrm{D}_{\text {frio }}-\mathrm{D}_{\text {forma }}}{\mathrm{D}_{\text {forma }}} \cdot 100$

Onde:

$\mathrm{Pf}=$ perda de forma circular $(\%)$;

$\mathrm{D}_{\text {forma }}=$ distância linear entre as extremidades do corpo de forma fixo na forma de vergamento $(\mathrm{cm})$; e

$\mathrm{D}_{\text {frio }}=$ distância linear entre as extremidades do corpo de prova em estado frio e desenformado (cm) (GATTO et al., 2008).

Adicionalmente, como parâmetro para a caracterização do comportamento da espécie, bem como da eficiência dos tratamentos, foi observado o número de tipos de defeitos $\left(\mathrm{N}_{\text {def }}^{o}\right)$ em cada corpo de prova após o ensaio de vergamento. Para tanto, foram considerados os tipos de defeitos verificados por Gatto et al. (2008), contabilizandose, por meio de análise visual, a ocorrência unitária de cada tipo.

A magnitude e a recorrência de defeitos do mesmo tipo, em cada corpo de prova, não foram consideradas como forma de inibir a subjetividade da análise visual e de acrescentar concisão aos resultados. Sendo assim, a variável $\mathrm{N}_{\text {def }}^{o}$ expressa a ideia de que, quanto menor o número médio de tipos de defeitos em cada tratamento, maior é seu potencial plasticizante e a consequente qualidade de vergamento.

Dessa forma, foi efetuada uma análise de variância fatorial para as variáveis $\Delta \mathrm{Tu}, \mathrm{Pe}, \mathrm{Pf}$ e $\mathrm{N}_{\text {def }}^{\mathrm{o}}$, tomando-se como fatores o tempo de tratamento em quatro níveis $(45,60,90$ e $120 \mathrm{~min})$ e o tipo de tratamento em dois níveis (cozimento e vaporização). A partir dos resultados da ANOVA fatorial, cada variável foi desdobrada por meio de testes de médias HSD de Tukey em 5\% de probabilidade de erro.

\section{Resultados e discussão}

Pode-se observar através da Tabela 2 que o fator tipo de tratamento apresentou efeito significativo para todas as variáveis avaliadas, com exceção da variável $\mathrm{N}_{\text {def. }}^{\mathrm{o}} \mathrm{O}$ tempo de tratamento mostrou-se com efeito significativo apenas para as variáveis de pressão de extremidade e perda de forma circular.

A interação tempo e tipo de tratamento para $\mathrm{N}_{\text {def }}^{\mathbf{o}}$ observada na Tabela 2 é mais bem evidenciada na Figura 3, com a apresentação do comportamento da variável em razão do tipo de tratamento e do tempo de tratamento, bem como a comparação entre as médias para os dois fatores.

Pode-se verificar que os valores médios de $\mathrm{N}_{\text {def }}^{\mathrm{r}}$ não diferiram significativamente entre tipos de tratamento para os tempos de $60 \mathrm{~min}$ e $120 \mathrm{~min}$. A 
diferenciação através do fator tipo de tratamento apenas apareceu para os tempos de $45 \mathrm{~min}$ e 90 $\min$.

Em adição, verificou-se que, para o tipo de tratamento de cozimento, o menor número de tipos de defeitos deu-se com 120 min de tratamento. Já para a vaporização, constatou-se comportamento inverso, sendo a menor média de defeitos por peça verificada no tratamento de menor tempo (45 $\mathrm{min}$ ).

Por meio da Figura 3, observa-se uma tendência à homogeneização dos efeitos do cozimento e da vaporização na incidência de defeitos de vergamento. Isso indica que, para a madeira avaliada, a variável $\mathrm{N}_{\text {def }}^{o}$ não representa um parâmetro aceitável para a qualificação ou diferenciação dos tipos de tratamento citados quanto à qualidade de vergamento. No entanto, ao contrário da vaporização, no tratamento de cozimento, verificou-se que, com o emprego de maiores tempos de tratamento $(>90 \mathrm{~min})$, foram evidenciados menores níveis de $\mathrm{N}_{\text {def. }}^{\mathbf{o}}$.

Assim, torna-se necessária a avaliação do comportamento de outras variáveis contidas no processo de vergamento visando à qualidade de processamento e produto final. Nesse contexto, a
Tabela 3 traz análises de variância para Pe, Pf e $\Delta \mathrm{Tu}$ em razão dos fatores tipo e tempo de tratamento.

Verificou-se na Tabela 3 que o tratamento de cozimento conferiu maior $\Delta \mathrm{Tu}$ à madeira de Eucalyptus grandis, o que também condicionou maiores valores de Pf. Em seu estudo, Kuljich, Cáceres e Hernández (2015) analisaram a perda de forma circular e o teor de umidade ao longo do tempo e verificaram retorno à forma inicial das peças vergadas mesmo após 60 dias e perda de umidade em até 30 dias de armazenamento. Com isso, autores concluíram que a variação no teor de umidade afeta de forma contundente a Pf, o que elucida que o desenvolvimento de tensões de retorno ocorre enquanto há movimentação de umidade na madeira.

Embora o mecanismo de impregnação de água oriundo de cada pré-tratamento empregado neste estudo seja distinto, verificou-se que a faixa de tempo em que os menores valores de Pf foram obtidos foi a mesma para o tratamento de vaporização e cozimento, a qual está entre $60 \mathrm{~min}$ e $90 \mathrm{~min}$.

Tabela 2 - Análise de variância fatorial para as variáveis analisadas em função do tipo e tempo de tratamento

\begin{tabular}{clcccc}
\hline Variável & Fonte de variação & SQ & Gl & QM & Valor F \\
\hline \multirow{3}{*}{$\mathrm{N}_{\text {def }}^{\text {}}$} & A: Tempo de Tratamento & 3,44 & 3,00 & 1,15 & $1,15^{\mathrm{ns}}$ \\
& B: Tipo de Tratamento & 2,81 & 1,00 & 2,81 & $2,83^{\mathrm{ns}}$ \\
& A x B & 8,44 & 3,00 & 2,81 & $2,83^{*}$ \\
& Resíduos & 71,50 & 72,00 & 0,99 & - \\
\hline \multirow{5}{*}{$\Delta \mathrm{Tu}$} & A: Tempo de Tratamento & 78,24 & 3,00 & 26,08 & $0,92^{\mathrm{ns}}$ \\
& B: Tipo de Tratamento & $1.941,67$ & 1,00 & $1.941,67$ & $68,13^{*}$ \\
& A x B & 145,82 & 3,00 & 48,61 & $1,71^{\mathrm{ns}}$ \\
& Resíduos & $1.966,35$ & 69,00 & 28,50 & - \\
\hline \multirow{3}{*}{$\mathrm{Pe}$} & A: Tempo de Tratamento & $1.124,27$ & 3,00 & 374,76 & $5,27^{*}$ \\
& B: Tipo de Tratamento & $1.967,61$ & 1,00 & $1.967,61$ & $27,69^{*}$ \\
& A x B & 435,18 & 3,00 & 145,06 & $2,04^{\mathrm{ns}}$ \\
& Resíduos & $5.116,73$ & 72,00 & 71,07 & - \\
\hline & A: Tempo de Tratamento & 20,51 & 3,00 & 6,84 & $4,95^{*}$ \\
& B: Tipo de Tratamento & 9,34 & 1,00 & 9,34 & $6,77^{*}$ \\
& A x B & 4,08 & 3,00 & 1,36 & $0,98^{\mathrm{ns}}$ \\
& Resíduos & 85,59 & 62,00 & 1,38 & - \\
\hline
\end{tabular}

Nota: Legenda:

$\mathrm{N}_{\text {def }}^{\circ}=$ número de defeitos;

$\Delta \mathrm{Tu}=$ variação de teor de umidade (\%);

$\mathrm{Pe}=$ pressão de extremidade $\left(\mathrm{kgf} . \mathrm{cm}^{-2}\right)$;

$\mathrm{Pf}=$ perda de forma circular (\%);

$\mathrm{SQ}=$ soma dos quadrados;

$\mathrm{Gl}=$ graus de liberdade;

$\mathrm{QM}=$ quadrado médio;

$\mathrm{F}=$ valor de $\mathrm{F}$ calculado;

* = significativo em $5 \%$ de probabilidade de erro; e

${ }^{\text {ns }}=$ não significativo. 
Figura 3 - Número de defeitos em função do tempo e do tipo de tratamento

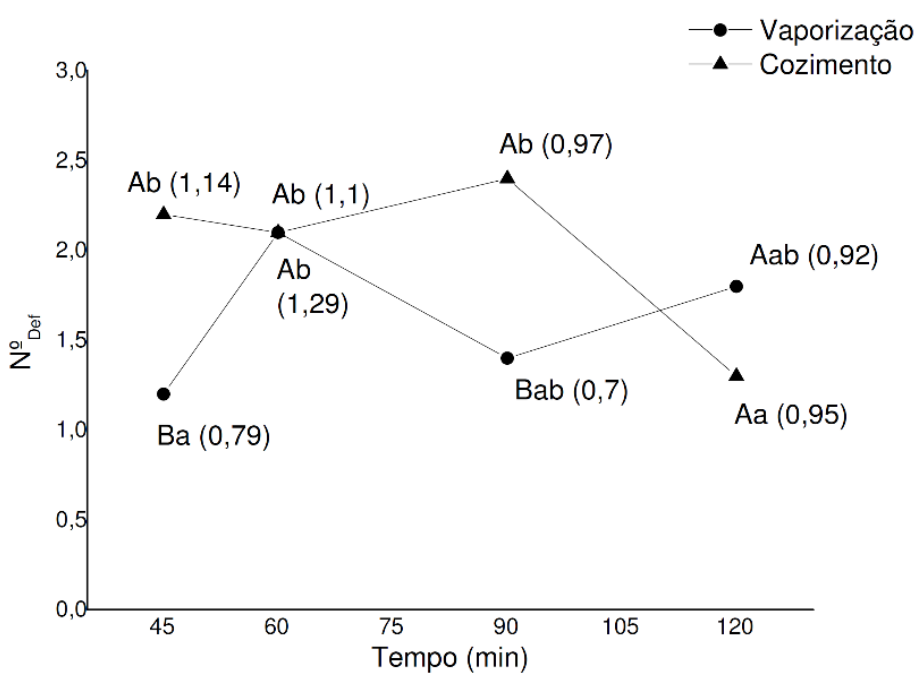

Nota: *médias com letras iguais maiúsculas verticalmente e minúsculas horizontalmente não apresentam diferença significativa em $5 \%$ de probabilidade de erro. Valores entre parênteses representam os desvios padrão.

Tabela 3 - Descrição das variáveis de estudo em razão dos fatores tipo e tempo de tratamento

\begin{tabular}{|c|c|c|c|c|c|}
\hline & \multicolumn{4}{|c|}{ Tempo de tratamento (min) } & \multirow{2}{*}{ Valor $\mathbf{F}$} \\
\hline & 45 & 60 & 90 & 120 & \\
\hline \multirow[t]{3}{*}{$\begin{array}{c}\text { Pe }\left(\mathrm{kgf.cm}{ }^{-2}\right) \\
\operatorname{Pf}(\%)\end{array}$} & $\begin{array}{c}51,16 \mathrm{ab} \\
5,35 \mathrm{~b}\end{array}$ & $\begin{array}{l}47,88 \mathrm{a} \\
4,48 \mathrm{ab}\end{array}$ & $\begin{array}{c}55,12 \mathrm{ab} \\
3,85 \mathrm{a}\end{array}$ & $\begin{array}{c}57,7 \mathrm{~b} \\
4,58 \mathrm{ab}\end{array}$ & $\begin{array}{l}3,79^{*} \\
4,26^{*}\end{array}$ \\
\hline & \multicolumn{4}{|c|}{ Tipo de tratamento } & \\
\hline & \multicolumn{2}{|c|}{ Vaporização } & \multicolumn{2}{|c|}{ Cozimento } & \\
\hline $\begin{array}{c}\text { Pe }\left(\mathrm{kgf.cm}{ }^{-2}\right) \\
\operatorname{Pf}(\%) \\
\Delta \mathrm{Tu}(\%)\end{array}$ & \multicolumn{2}{|c|}{$\begin{array}{c}48,01 \mathrm{a} \\
4,25 \mathrm{a} \\
17,05 \mathrm{a}\end{array}$} & \multicolumn{2}{|c|}{$\begin{array}{c}57,68 \mathrm{~b} \\
4,96 \mathrm{~b} \\
26,95 \mathrm{~b}\end{array}$} & $\begin{array}{c}22,99 * \\
5,55 * \\
64,70 *\end{array}$ \\
\hline
\end{tabular}

Nota: Legenda:

$\Delta T u=$ variação de teor de umidade;

$\mathrm{Pe}=$ pressão de extremidade;

$\mathrm{Pf}=$ perda de forma circular;

* = significativo em $5 \%$ de probabilidade de erro; e

Médias seguidas de letras iguais não apresentam diferença significativa de acordo com teste HSD de Tukey.

Através da Tabela 3 verifica-se maior $\Delta \mathrm{Tu}$ para o cozimento, o que indica melhor adequação desse tratamento para situações que exijam aumento expressivo no teor de umidade. Por outro lado, nos casos em que a adição de umidade não se apresenta como fator altamente desejável, ou para madeiras com elevado teor de umidade inicial, a vaporização é indicada, por apresentar melhor eficiência energética.

Gasparik e Gaff (2013), analisando a variação do teor de umidade ao longo do tratamento de plasticização por micro-ondas da madeira de Fagus sylvatica aos 75 anos de idade, concluíram que o teor de umidade de $40 \%$ foi considerado ótimo para o vergamento da madeira. Dessa forma, a escolha do tratamento com base em seu potencial de adição de umidade é aconselhável, desde que sejam conhecidos parâmetros como o teor de umidade alvo (teor de umidade final) e o teor de umidade atual (teor de umidade inicial) da madeira.

Como já citado, comparando-se com o valor obtido para a vaporização, a Pf apresentou-se estatisticamente maior para o tipo de tratamento de cozimento. Entretanto, quando considerado o fator tempo de tratamento, a Pf não apresentou uma tendência clara, embora com diferença estatística entre os tempos. Foi verificado o maior valor médio para o menor tempo (45 min), e o menor valor médio para um tempo intermediário (90 $\min )$.

Na análise da pressão de extremidade, pôde-se verificar diferença estatística entre tempos e tipos de tratamento. O tipo de tratamento de cozimento apresentou valor significativamente mais elevado do que na vaporização. Esse fato é explicado pelo maior conteúdo de umidade no interior da peça

174 Peres, M. L.; Delucis, R. de A.; Gatto, D. A.; Beltrame, R. 
durante o cozimento. No momento do vergamento, acompanhando a deformação da peça, são desenvolvidas tensões internas, as quais ocasionam a movimentação da água contida no interior da madeira. Com isso, há maior desenvolvimento de forças de reação em toda a peça submetida ao esforço mecânico.

A Figura 4 ilustra o comportamento das variáveis em estudo em razão dos pré-tratamentos empregados e dá conta de que o tratamento de cozimento conferiu maior valor para todas as variáveis. Uma maior $\Delta T u$ pode ser apreciável em circunstâncias em que é baixo o teor de umidade inicial da peça a ser vergada. Assim, é possível afirmar que o tratamento de vaporização proporciona melhor qualidade de vergamento, além de demandar menos energia calorífica.

Zhao et al. (2012) estudaram o módulo de relaxamento da madeira como forma de representação das tensões internas desenvolvidas na peça em oposição à deformação. Os autores concluíram que o referido módulo decresce com o aumento da temperatura e do conteúdo de umidade. Sendo assim, quanto maior é a liberação de água durante o processo de estabilização térmica e higroscópica da madeira, mais pronunciado é o desenvolvimento das referidas tensões presentemente descritas pela variável Pf. Tal relação explica a verificação de menores valores de Pf para a vaporização, uma vez que a deformação plástica é proporcionada com teores de umidade mais baixos, além de elucidar a influência do conteúdo de umidade na perda de forma circular.

As variáveis de Pf e Pe apresentaram valores maiores para o cozimento. Tal comportamento é explicado no estudo de Ishimaru, Oshima e Iida (2001), em que foram avaliadas propriedades mecânicas da madeira em condicionamentos de umidade por adsorção e desorção. Os autores citam que o relaxamento de tensões internas é maior para condicionamentos por absorção de umidade, comparado com a desorção, corroborando a observância do valor médio de Pf superior verificado para o cozimento. Além disso, verificaram um aumento na resistência e na elasticidade da madeira nos estágios iniciais de desorção, isto é, a perda de umidade também observada como processo subsequente ao vergamento. Essa constatação fundamenta os maiores valores de $\mathrm{Pe}$ encontrados para o cozimento.

Considerando a complexidade do processo de vergamento, a variabilidade das espécies de madeira e a diversidade dos métodos de plasticização, os resultados obtidos podem ser considerados válidos para a situação em questão. Estudos das variáveis inerentes à madeira e ao processo de vergamento devem continuar sendo desenvolvidos, com o objetivo de particularizar as situações e otimizar o processo para fins industriais. Assim, peças de madeira vergada, atualmente produzidas por empirismo, terão cada vez maior confiabilidade para a aplicação em grande escala.

Figura 4 - Comportamento das variáveis de estudo em relação aos tipos de tratamento. Valores entre parênteses representam os desvios padrão

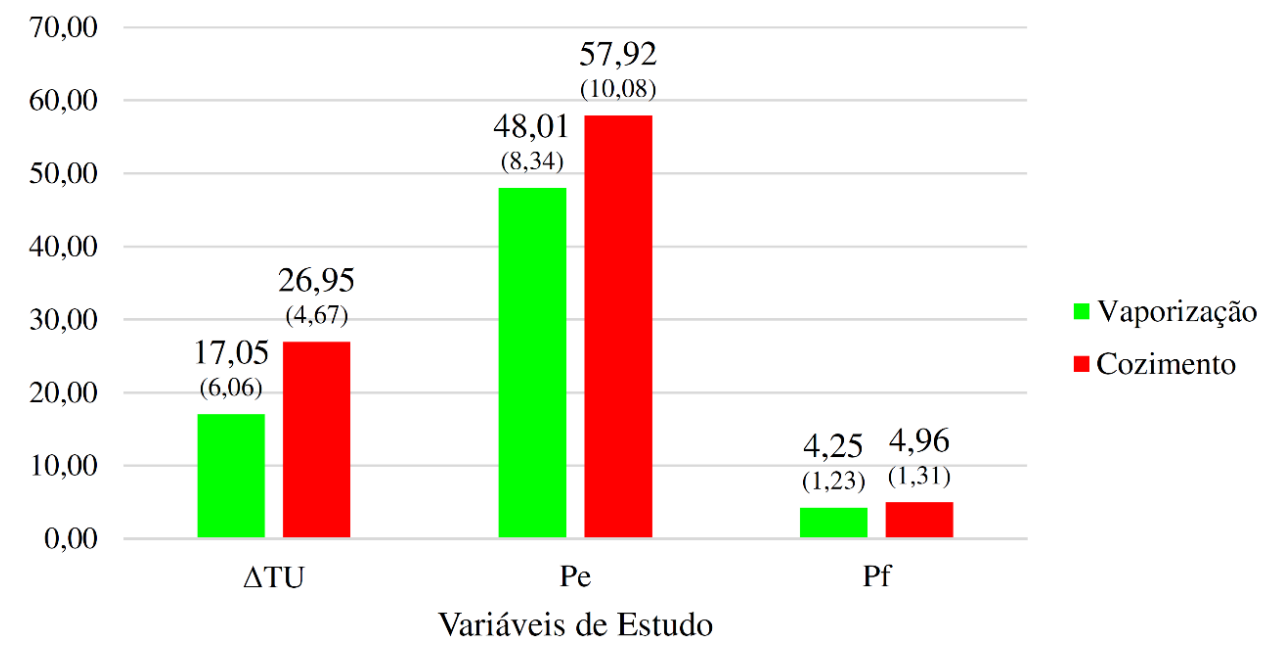




\section{Conclusões}

De maneira geral, os parâmetros utilizados para a avaliação da qualidade das peças vergadas apresentaram comportamentos sinérgicos.

A madeira utilizada pode ser considerada aceitável para vergamentos sob raios de curvatura inferiores ao adotado no presente trabalho.

O número de defeitos por peça não se apresentou como variável adequada para a caracterização do pré-tratamento quanto à qualidade de vergamento.

O fator tempo de tratamento, considerando-se tempos acima de $45 \mathrm{~min}$, não exerce efeito na qualidade de vergamento.

A vaporização mostrou-se um pré-tratamento mais adequado, em detrimento do cozimento.

\section{Referências}

\section{AMERICAN SOCIETY FOR TESTING AND MATERIALS. Standard Test Methods For Small Clear Specimens of Timber - ASTM D143-94. West Conshohocken, 2007.}

AYARKWA, J.; OWUSU, F. W.; APPIAH, J. K. Steam Bending Qualities of Eight Timer Species of Ghana. Ghana Journal of Forestry, v. 27, n. 2, p. 12-22, 2011.

DAIAN, G.; OZARSKA, B. Impact of Sapwood and the Properties and Market Utilization of Plantation and Young Hardwoods: determination of minimum radius of bending curvature of heartwood and sapwood from plantation and young hardwood species using microwave wood bending technology (PART B). Melbourne: Forest \& Wood Products Australia (FWPA), 2010.

GAŠPARÍK, M.; GAFF, M. Changes in Temperature and Moisture Content in Beech Wood Plasticized by Microwave Heating. BioResources, v. 8, n. 3, p. 3372-3384, 2013.
GATTO, D. A. et al. Características Tecnológicas das Madeiras de Luehea divaricata, Carya illinoinensise e Platanus $x$ acerifolia Quando Submetidas ao Vergamento. Ciência Florestal, v. 18, n. 1, p. 121-131, 2008.

HWANG, K. et al. Bending Quality of Main Korea Wood Species. Wood Research, v. 89, n. 3 p. 6-10, 2002.

ISHIMARU, Y.; OSHIMA, K.; IIDA, I. Changes in the Mechanical Properties of Wood During a Period of Moisture Conditioning. Journal of Wood Science, v. 47, n. 4, p. 254-261, 2001.

KULJICH, S.; CÁCERES, C.; HERNÁNDEZ, R. Steam-Bending Properties of Seven Poplar Hybrid Clones. International Journal of Material Forming, v. 8, n. 1, p. 67-72, 2015.

PERES, M. L. Qualidade da Madeira Vergada de Eucalyptus grandis Hill Ex Maiden. Pelotas, 2011. 57 f. Trabalho de Conclusão de Curso (Graduação) - Faculdade de Engenharia Industrial Madeireira, Universidade Federal de Pelotas, Pelotas, 2011.

PERES, M. L. et al. Vergamento de Madeira Sólida: qualidade de processo e matéria-prima. Nativa, v. 1, n. 1, p. 56-61, 2013.

SCHLEINING, L. The Complete Manual of Wood Bending: milled, laminated, and steam-bent work. Fresno: Linden Publishing, 2001.

VON ZUBEN, D. L. Desenvolvimento de Processo de Fabricação Por Vergamento de Madeira Maciça. Campinas, 2010. 46 f. Trabalho de Conclusão de Curso (Graduação) - Faculdade de Engenharia Mecânica, Universidade São Francisco, Campinas, 2010.

ZHAO, Y. K. et al. Viscoelastic Properties of Wood From Chinese-Fir and Poplar Plantations. Forestry Studies in China, v. 14, n. 2, p. 107$111,2012$. 
Matheus Lemos Peres

Centro de Desenvolvimento Tecnológico | Universidade Federal de Pelotas | Rua Conde de Porto Alegre, 793, Centro | Pelotas - RS Brasil | CEP 96010-290 | Tel.: (53) 3921-1265 | E-mail: matheusldeperes@gmail.com

Rafael de Avila Delucis

Escola de Engenharia | Universidade Federal do Rio Grande do Sul | Av. Bento Gonçalves, 9500, Setor 4, Prédio 74, Agronomia | Porto Alegre - RS - Brasil | CEP 91509-900 | Tel.: (51) 3308-9419 | E-mail: r.delucis@hotmail.com

\section{Darci Alberto Gatto}

Centro de Engenharias | Universidade Federal de Pelotas | E-mail: darcigatto@yahoo.com

\section{Rafael Beltrame}

Centro de Engenharias | Universidade Federal de Pelotas | E-mail: beltrame.rafael@yahoo.com.br

\section{Revista Ambiente Construído}

Associação Nacional de Tecnologia do Ambiente Construído

Av. Osvaldo Aranha, $99-3^{\circ}$ andar, Centro

Porto Alegre - RS - Brasil

CEP $90035-190$

Telefone: +55 (51) 3308-4084

Fax: +55 (51) 3308-4054

www.seer.ufrgs.br/ambienteconstruido

E-mail: ambienteconstruido@ufrgs.br 